\title{
The Role of SNCA Rep1 Microsatellite in Parkinson's Disease Progression
}

\author{
Lucia Corrado a, Fabiola De Marchi $^{\text {b, Sara Tunesi }}{ }^{\text {c,d }}$, Gaia Oggioni ${ }^{\text {b,e }}$, Miryam Carecchio ${ }^{\text {b, }}$ \\ Luca Magistrelli b, Silvana Tesei ${ }^{\text {, }}$ Giulio Riboldazzi e, Alessio Di Fonzo ${ }^{\text {g, Clarissa Locci a }}$, \\ Ilaria Trezzi g, Roberta Zangaglia ${ }^{\text {h }}$, Cristina Cereda i, Sandra D'Alfonso a, Corrado Magnani ${ }^{\text {c, }}$ \\ Comi Giacomo Pietro g, Giorgio Bono e, Claudio Pacchetti h, Roberto Cantello b, \\ Stefano Goldwurm ${ }^{\mathrm{f}}$ and Cristoforo Comi ${ }^{\mathrm{b}}$
}

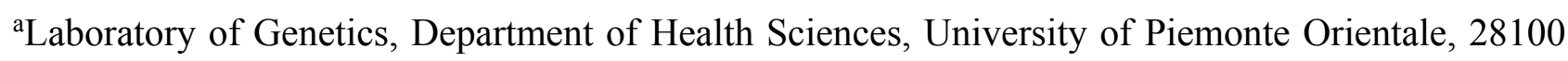
Novara, Italy

${ }^{b}$ Department of Translational Medicine, Section of Neurology, University of Piemonte Orientale, 28100 Novara, Italy

${ }^{\mathrm{c}}$ Department of Translational Medicine, Unit of Medical Statistics and Cancer Epidemiology, University of Piemonte Orientale, 28100 Novara, Italy

${ }^{\mathrm{d}}$ Center for Cancer Epidemiology and Prevention (CPO), University Hospital "Città della Salute e della Scienza di Torino", 10126 Turin, Italy

eParkinson's Disease and Movement Disorders Center, Ospedale di Circolo Fondazione Macchi, University of Insubria, Varese, Italy

fParkinson Institute, ASST Gaetano Pini-CTO (formerly ICP), 21100 Milan, Italy

'IRCCS Foundation Ca' Granda Ospedale Maggiore Policlinico, Dino Ferrari Center, Neuroscience Section, Department of Pathophysiology and Transplantation, University of Milan, 20122 Milan, Italy

hParkinson's Disease and Movement Disorders Unit, C. Mondino National Institute of Neurology Foundation, IRCCS, 27100 Pavia, Italy

${ }^{\mathrm{i}}$ Genomic and post-Genomic Center, C. Mondino National Institute of Neurology Foundation, IRCCS, 27100 Pavia, Italy 
*Corresponding author: Cristoforo Comi, $\mathrm{MD}, \mathrm{PhD}$

Department of Translational Medicine

University of Piemonte Orientale

Via Solaroli 17 - 28100 Novara, Italy.

Phone number: +39.0321 .3733965$

Email address: comi@med.uniupo.it

ORCID number: http://orcid.org/0000-0002-6862-9468

Funding agencies: This research was supported by the University of Piemonte Orientale, "Fondi di Ricerca Dipartimentale” and by AriSLA (REPEATALS grant).

Relevant conflicts of interests/financial disclosures: The authors report no conflict of interest. 


\begin{abstract}
$\alpha$-synuclein is a constituent of Lewy bodies and mutations of its gene cause familial PD. A previous study showed that a variant of $\alpha$-synuclein gene $(S N C A)$, namely the $263 \mathrm{bp}$ allele of Rep1 was associated to faster motor progression in PD. On the contrary, a recent report failed to detect a detrimental effect of Rep1 263 on both motor and cognitive outcomes in PD. Aim of this study was to evaluate the influence of the Rep1 variants on disease progression in Parkinson's Disease (PD) patients. We recruited and genotyped for $S N C A$-Rep1 426 PD patients with age at onset $\geq 40$ years and disease duration $\geq 4$ years. We then analyzed frequency and time of occurrence of wearing-off, dyskinesia, freezing of gait, visual hallucinations and dementia. SNCA Rep1 263 carriers showed increased risk of both dementia $(\mathrm{HR}=3.03)$ and visual hallucinations $(\mathrm{HR}=2.69)$ compared to 263 non-carriers. In conclusion, SNCA Rep 1263 allele is associated to a worst cognitive outcome in PD.
\end{abstract}

Keywords: dementia; hallucinations; genetics 


\section{Introduction}

Parkinson's disease (PD) is the second most frequent neurodegenerative disease [1], and is clinically defined by the presence of resting tremor, rigidity, and bradykinesia [2]. Nonetheless, non-motor features such as mood deflection, anosmia, and sleep disturbances can even pre-date motor impairment in PD [3]. As disease progresses, further disabling symptoms appear, such as posture and gait impairment on the motor side, and cognitive decline and hallucinations in the nonmotor domain $[4,5]$.

PD neuropathology is characterized by the loss of dopaminergic neurons and the presence of Lewy bodies (LBs) in surviving neurons [6,7]. LBs contain fibrils composed of alpha-synuclein, a small protein involved in synaptic vesicle trafficking and neurotransmitter release [7] but also in more widespread functions, including the triggering neuroinflammatory processes [8]. Duplication and triplication of alpha-synuclein gene $(S N C A)$ cause dominant early-onset PD, suggesting that overexpression of wild-type alpha-synuclein is sufficient to cause the disease $[9,10,11]$. The amount of alpha-synuclein is relevant in sporadic disease as well, since its expression is higher in brain tissue from PD patients compared to control tissue [12]. Furthermore, genome wide association studies revealed that $S N C A$ variations are associated with sporadic PD development [13].

Variations in the complex microsatellite D4S3481 (called Rep-1), located approximately 10kb upstream of the translational start of $S N C A$, have been reported to increase PD risk. SNCA Rep1 is essentially triallelic (259, 261, and 263 base pairs in length) and a meta-analysis of association studies showed higher frequency of $263 \mathrm{bp}$ allele in cases than in controls [14]. Furthermore, the $259 \mathrm{bp}$ allele was found to be associated to a decreased risk of PD, being more frequent in controls than in cases, whereas no relevant effect was observed for the $261 \mathrm{bp}$ allele [14]. To date, data on the role of SNCA common variants in PD progression are conflicting. Ritz et al. analyzed 232 PD patients and found that the risk of faster motor decline was 4-fold increased in carriers of the Rep-1 $263 b p$ promoter variant [15]. Thereafter, a study in which outcome was measured in terms of life expectancy, did not detect any association between $S N C A$ Rep-1 genotypes and risk of death in 
6154 PD cases [16]. More recently, Markopoulou et al. [17] analyzed the correlation between SNCA Rep-1 genotypes and both motor and cognitive outcomes in a large cohort of PD patients. Surprisingly, they found an opposite role of the microsatellite variants, with shorter alleles providing worst outcomes.

On this background, the aim of our study was to investigate whether SNCA Rep-1 may influence the progression of Parkinson's disease in a cohort of Italian PD patients, clinically characterized trough the collection of solid and reliable milestones of disease evolution [Williams-Gray CH, Mason SL, Evans JR, Foltynie T, Brayne C, Robbins TW, Barker RA. The CamPaIGN study of Parkinson's disease: 10-year outlook in an incident population-based cohort. J Neurol Neurosurg Psychiatry. 2013 Nov;84(11):1258-64..

\section{Patients and Methods}

\subsection{Patients}

426 patients (249 males) with PD [2] were enrolled according to the following inclusion criteria: age at onset $\geq 40$ years, longitudinal follow-up $\geq 4$ years. All patients were of Italian origin and were enrolled at the following Movement Disorders Centers: 1) University of Piemonte Orientale, Novara, 2) University of Insubria, Varese, 3) IRCCS Foundation Ca' Granda Ospedale Maggiore Policlinico, Dino Ferrari Center, Neuroscience Section, University of Milan 4) C. Mondino National Institute of Neurology Foundation, IRCCS, Pavia 5) Parkinson Institute, ASST Gaetano Pini-CTO, Milan. The Parkinson Institute cohort (144 patients) had been previously analyzed in both a case-control study assessing the role of SNCA variants in PD susceptibility [18] and in the collaborative GEO-PD study on survival [16].

Patients who had at least one $1^{\text {st }}$ or $2^{\text {nd }}$ degree relative with a diagnosis of primary parkinsonism, and/or age at onset $\leq 50$ years and/or peculiar clinical features had been previously analyzed to exclude pathogenic mutations on known PD-genes (SNCA, LRRK2, Parkin, PINK1, DJ-1) according to EFNS guidelines [19]. 
This study was approved by the local Ethics Committee. Patients were included in the study after having read and signed an informed consent form for research purpose.

\subsection{Assessment of $P D$ progression}

All recruited patients had been longitudinally followed-up by neurologists expert in movement disorders at each center. The mean follow up duration was 11.3 years. Clinical records were retrospectively analyzed taking into consideration only variables that had been assessed in each center. Agreement was found on the following parameters: gender, age at onset, disease duration, and family history. Furthermore, the presence and time of onset of the following motor and nonmotor complications was recorded: wearing-off, dyskinesia, freezing of gait, visual hallucinations and dementia. Dementia was diagnosed according to MDS criteria [20].

\subsection{Determination of $S N C A$ Variants}

Genomic DNA extraction was performed from peripheral blood using standard procedures. We analyzed the microsatellite $S N C A$ Rep1 in the entire cohort (426 subjects). The $S N C A$ Rep1 region was PCR amplified from genomic DNA using the primer couple: 5'GACTGGCCCAAGATTAACCA-3' (fluorescently labeled with 6-FAM) and 5'CCTGGCATATTTGATTGCAA-3' PCR products were resolved by capillary electrophoresis on an ABI-3130XL DNA Analyzer (Applied Biosystem, Foster City, CA, USA) using GeenScan-500 ROX (Applied Biosystem) as molecular weight marker Allelic sizes were assessed using the GeneMapper 4.0 software.

To confirm the accuracy of the genotyping method, we sequenced several individuals representative of each genotype as standard samples.

\subsection{Statistical Analysis}

Allelic distribution was assessed for Hardy-Weinberg equilibrium (HWE) with Fisher's exact test. 
SNCA Rep1 was analyzed using a dominant genetic model (e.g. subjects 263-carriers vs 263-non carriers) as previously described [15]

Continuous variables were reported as median and $25^{\text {th }}$ and $75^{\text {th }}$ percentile, categorical variables were presented as frequencies (counts) and percentages. Allelic frequencies were compared with the $\chi 2$ test, differences between age at onset and disease duration were assessed with MannWhitney U Test.

The influence of SNCA variant carrier status on disease natural history was investigated performing time-to-event analysis: wearing-off, dyskinesia, freezing of gait, visual hallucinations and dementia were considered as separate outcomes. Every patient contributed time of observation from disease onset to complication under study or the last assessment. Individual analyses were performed for each complication under study and cumulative incidences were estimated using the Kaplan-Meier (KM) methods. Log-rank test was used to examine univariable association with outcome. Multivariable Cox's proportional hazards regression model was fitted to obtain the hazard ratio (HR) for $S N C A$ variant carrier status adjusted by gender, age at PD onset and recruitment center.

Proportional hazard $(\mathrm{PH})$ model was assessed by regression scaled Schoenfeld residuals against the $\log$ time. All $\mathrm{P}$ values are 2-tailed and the significance cut-off was $\mathrm{P}<0.05$. Statistical analysis was performed using STATA v14.

\section{Results}

Demographic and clinical features of the 426 PD patients are summarized in Table 1.

Observed frequencies of SNCA Rep1 genotypes were in HWE ( $\mathrm{p}=0.19)($ Supplementary Table 1).

Disease duration did not differ significantly between Rep1 263 carriers and 263 non-carriers. On the contrary, the former group showed significantly earlier age at onset compared to the latter ( $p=0.016$, and $\mathrm{p}=0.088$ respectively, Mann-Whitney U Test; supplementary Table 2).

To compare the probability to develop each complication over time in 263 carriers vs 263 noncarriers, we performed a Kaplan-Meyer survival analysis. (Figure 1 and Supplementary Figure 1). At ten years from onset, cumulative incidence of dementia was higher in 263 carriers than in 263 
non-carriers (26.39 vs $13.70 \%$, log-rank test $\mathrm{p}=0.001$; Table 2$)$. Similar findings were also observed for visual hallucinations and wearing-off (24.48 vs $15.01 \%$ and 65.33 vs $53.02 \%$, log-rank test $\mathrm{p}=0.0037$ and $\mathrm{p}=0.028$ respectively; Table 2 ).

Finally, after applying a multivariable Cox regression model adjusting by gender, age at PD onset and recruitment center, a statistically significant difference persisted for dementia and visual hallucinations but not for wearing-off. In detail, we found that 263 carriers had 3.03 higher risk of dementia, 2.69 higher risk of visual hallucinations, and 1.26 higher risk of wearing-off compared to 263 non-carriers $\quad(\mathrm{p}<0.001, \quad=0.001, \quad$ an

0.236 respectively; Table $3)$. 
Table 1. Demographic and clinical features of study population.

\begin{tabular}{|l|c|}
\hline & $\mathbf{n}=\mathbf{4 2 6}$ \\
\hline Male, $\mathbf{n}, \mathbf{( \% )}$ & $249(58.5 \%)$ \\
\hline Age at onset, median $\left(25^{\text {th }}-75^{\text {th }}\right)$ & $62(55-68)$ \\
\hline Age at assessment, median $\left(25^{\text {th }}-75^{\text {th }}\right)$ & $74.0(68-79)$ \\
\hline Disease duration, median $\left(25^{\text {th }}-75^{\text {th }}\right)$ & $11.0(8-14)$ \\
\hline Familiy history, $\mathbf{n},(\%)$ & $81(19.0 \%)$ \\
\hline Wearing-off, $\mathbf{n}, \mathbf{( \% )}$ & $231(54.2 \%)$ \\
\hline Dyskinesia, $\mathbf{n}, \mathbf{( \% )}$ & $196(46.0 \%)$ \\
\hline Freezing of gait, $\mathbf{n}, \mathbf{( \% )}$ & $169(39.7 \%)$ \\
\hline Visual hallucinations, $\mathbf{n},(\%)$ & $85(20.0 \%)$ \\
\hline Dementia, $\mathbf{n}, \mathbf{( \% )}$ & $77(18.1 \%)$ \\
\hline
\end{tabular}

Table 2. Probability of complications at $\mathbf{1 0}$ years from disease onset.

\begin{tabular}{|c|c|c|c|}
\hline & $\begin{array}{c}\text { Rep 1 263 carriers } \\
\% \text { at } 10 \text { years }(95 \% \mathrm{CI})\end{array}$ & $\begin{array}{c}\text { Rep 1 263 non carriers } \\
\% \text { at } 10 \text { years }(95 \% \mathrm{CI})\end{array}$ & $\mathrm{P}^{*}$ \\
\hline Wearing-off & $\begin{array}{c}65.33 \\
(50.03-80.14)\end{array}$ & $\begin{array}{c}53.02 \\
(47.47-58.59)\end{array}$ & 0.028 \\
\hline Dyskinesia & $\begin{array}{c}44.10 \\
(29.81-61.55)\end{array}$ & $\begin{array}{c}44.05 \\
(38.67-49.81)\end{array}$ & 0.360 \\
\hline $\begin{array}{c}\text { Freezing of } \\
\text { gait }\end{array}$ & $\begin{array}{c}33.19 \\
(21.07-49.72)\end{array}$ & $\begin{array}{c}34.25 \\
(29.18-39.92)\end{array}$ & 0.621 \\
\hline $\begin{array}{c}\text { Visual } \\
\text { hallucinations }\end{array}$ & $\begin{array}{c}24.48 \\
(13.88-40.97)\end{array}$ & 15.01 & 0.004 \\
\hline Dementia & 26.39 & $(11.50-19.47)$ & 0.001 \\
\hline
\end{tabular}

*Log-rank test 
Table 3 Multivariable Cox's regression model on all study population.

\begin{tabular}{|c|c|c|}
\hline & HR $(\mathbf{9 5} \% \mathbf{C I}) *$ & P \\
\hline Wearing-off & $1.26(0.86-1.86)$ & 0.236 \\
\hline Dyskinesia & $0.94(0.62-1.45)$ & 0.794 \\
\hline Freezing of gait & $0.97(0.61-1.55)$ & 0.901 \\
\hline Visual hallucinations & $2.69(1.57-4.60)$ & 0.001 \\
\hline Dementia & $3.03(1.74-5.30)$ & $<0.001$ \\
\hline
\end{tabular}

*Hazard Ratio (HR) of Rep 1263 carriers versus 263 non carriers.

Data are adjusted by gender, age at PD onset and recruitment center. 
Supplementary Table 1. Genotype distribution frequencies.

\begin{tabular}{|l|l|l|}
\hline & & \\
\hline & & $\mathbf{N}(\mathbf{\%}), \mathbf{n}=\mathbf{4 2 6}$ \\
\hline & & \\
\hline \multirow{5}{*}{ Rep 1 } & $257 / 259$ & $1(0.23 \%)$ \\
\cline { 2 - 3 } & $259 / 259$ & $22(5.16 \%)$ \\
\cline { 2 - 3 } & $259 / 261$ & $147(34.51 \%)$ \\
\cline { 2 - 3 } & $259 / 263$ & $7(1.64 \%)$ \\
\cline { 2 - 3 } & $261 / 261$ & $212(49.77 \%)$ \\
\cline { 2 - 3 } & $261 / 263$ & $35(8.22 \%)$ \\
\cline { 2 - 3 } & $263 / 263$ & $2(0.47 \%)$ \\
\hline
\end{tabular}


Supplementary Table 2. Age at PD onset and disease duration for 263 carriers and 263 noncarriers.

\begin{tabular}{|l|l|l|l|}
\hline & \multicolumn{3}{|c|}{$\mathbf{N}(\%), \mathbf{n = 4 2 6}$} \\
\hline & 263 carriers & 263 non carriers & P Mann-Whitney U Test \\
\hline $\begin{array}{l}\text { Age at PD } \\
\text { onset median }\left(25^{\text {th }}-75^{\text {th }}\right)\end{array}$ & 56 & 62 & 0.016 \\
\hline Disease duration & $(53-64)$ & $(55-68)$ & \\
& 12.5 & 11 & 0.088 \\
\hline
\end{tabular}


Figure 1. Kaplan-Meyer survival analysis of complications (dementia panel A; visual hallucinations panel B; wearing-off panel C). 


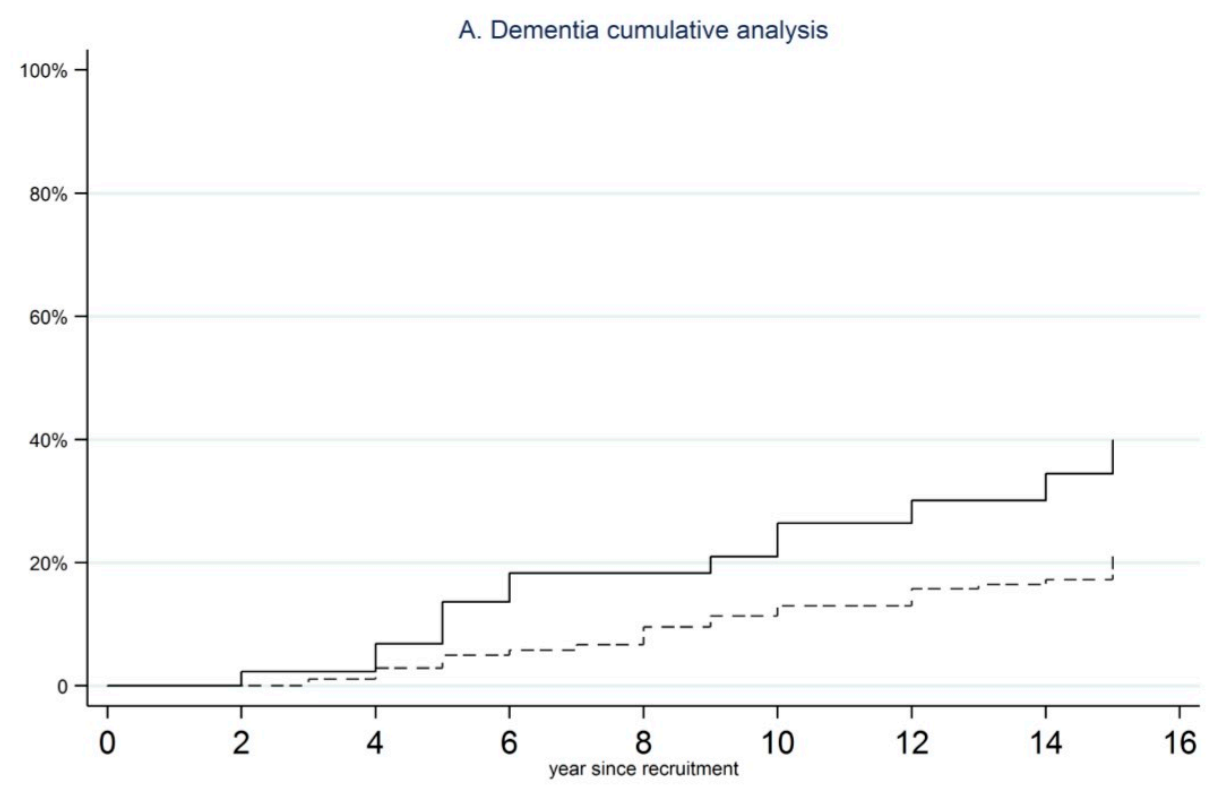

-- -- - 263 non-carriers 263 carriers

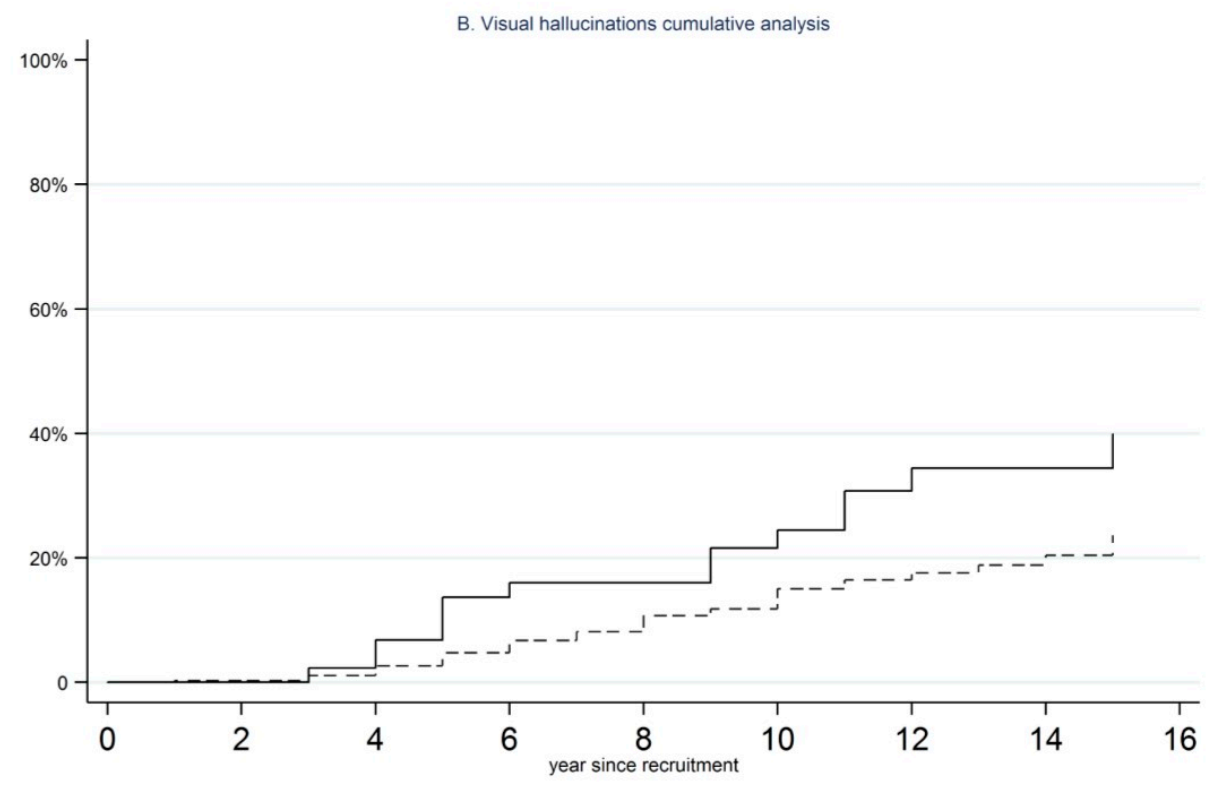

------263 non-carriers 263 carriers

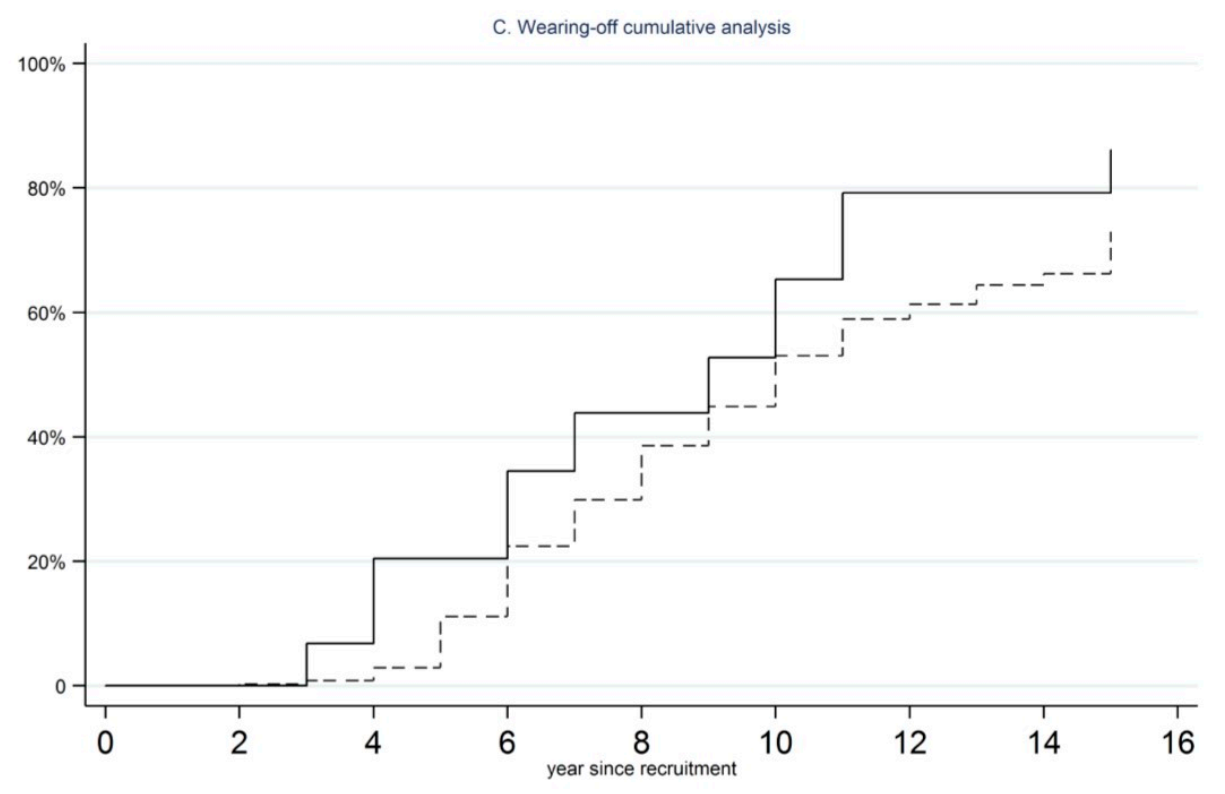

- - - - 263 non-carriers 263 carriers 
Supplementary Figure 1. Kaplan-Meyer survival analysis of complications (dyskinesia panel B; freezing of gait panel A). 

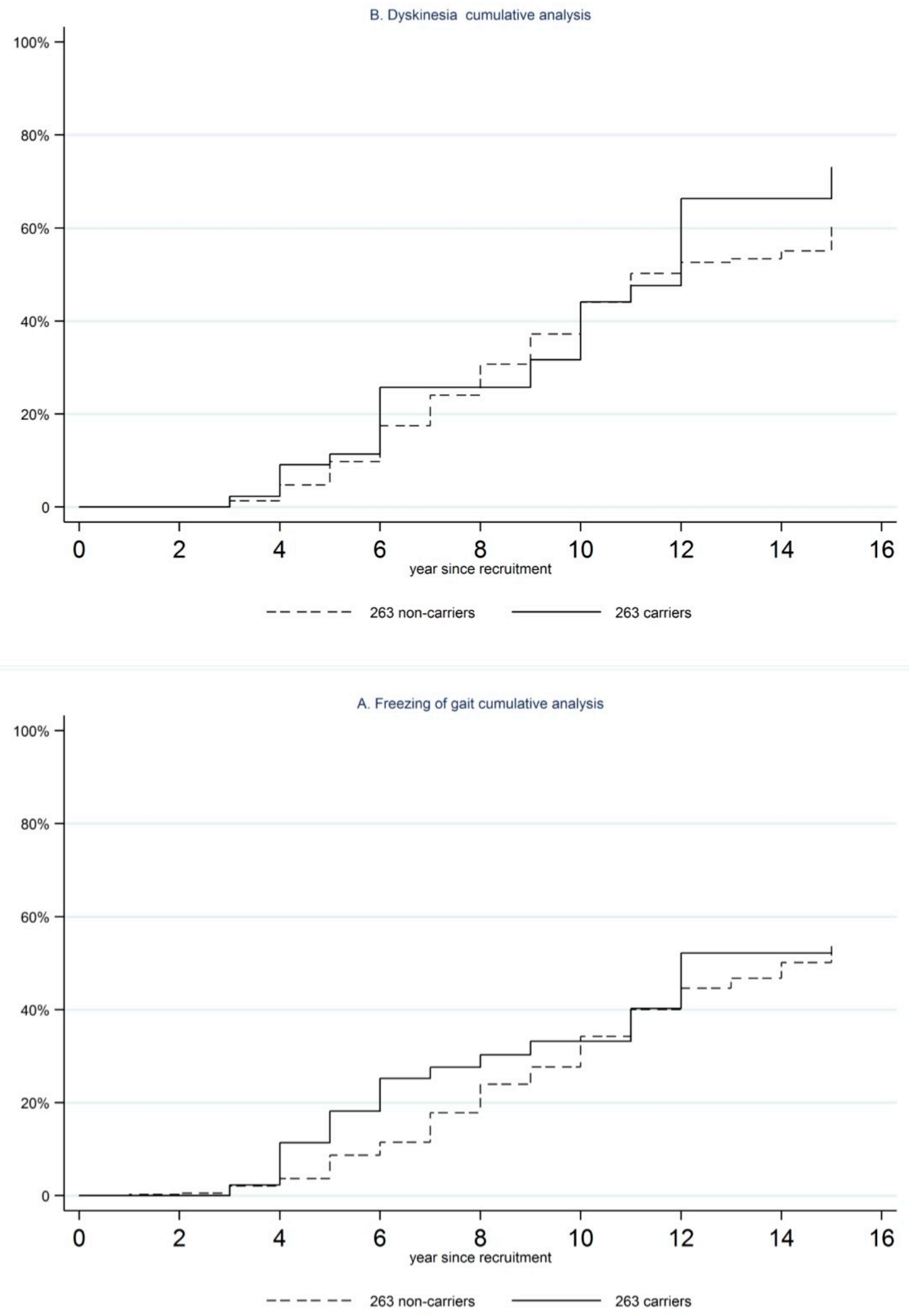


\section{Discussion}

This study showed for the first time that Rep1 polymorphic variants of SNCA influence non-motor progression in PD. PD patients who carry SNCA Rep1 263 allele have 3.03 increased risk of dementia and 2.69 increased risk of visual hallucinations compared to patients carrying shorter Rep1 variants. As regards motor progression, Rep1 263 carriers display slightly increased cumulative incidence of wearing-off, compared to 263 non-carriers, even though such difference was not statistically significant in multivariable regression.

To date, strong evidence supports a genetic component in PD susceptibility, whereas studies showing a relevant role for disease modifying genes are less represented $[21,22,23]$. This might be due, at least in part, to the difficulty in collecting data on disease evolution compared to recording disease development. Consistently, large collaborative studies aimed at tracking PD progression are ongoing [24, 25] In our study, we choose to include only patients with longitudinal follow-up and accurate information on the main motor and non-motor complications, putting together a very well characterized population of 426 patients.

The cognitive features of 263 carriers recalled that of familial PD caused by rare pathogenic SNCA multiplications, in which progression is faster compared to sporadic PD [26]. It is known that such mutations cause alpha-synuclein over-production through increased mRNA expression [27]. As regards the possible effect of the length of Rep1 microsatellite on alpha-synuclein expression, conflicting data were reported. Chiba-Falek et al. detected a 3-fold increase of alpha-synuclein expression with longer compared to shorter Rep1 alleles in a cellular model [28]. Furthermore, alpha-synuclein mRNA varied 1.7-fold in transgenic mice carrying the different Rep1 alleles [29]. These findings were further supported by Fuchs et al. who detected a correlation between protein level in blood and Rep1 genotypes [30]. On the contrary, a recent a report by Soldner et al. suggested that neither the deletion of the entire Rep1 repeat sequence nor the presence of the three different length alleles (including 263bp) were able to influence significantly SNCA expression. Nonetheless, the authors themselves underline that their findings might have been influenced by the 
cellular model analysed, which allows to detect only early events [31]. For such reasons, a robust biologic relationship between the 263 allele and PD progression still has to be confirmed

Our study is the first to explore the correlation between SNCA polymorphisms and PD progression in the European population. On the contrary, two Northern American studies, the first on 233 patients and the second on 1098 patients, were published in 2012 and 2014 respectively [14,15].

In the first study, a faster motor progression in PD patients carrying at least one Rep1 263 was reported [15]. As regards motor progression in Rep1 263 carriers, our findings display a trend similar to Ritz et al. [15], even though our data lose statistical significance in the multivariable regression model. Nonetheless, it should be noted that discrepancies might be related to substantial methodological differences. In fact, Ritz et al [15] studied a cohort of 233 PD patients for a relatively short follow-up (5.1 years) and measured motor progression with the motor section of UPDRS. This score: a) does not weigh the different motor signs, b) does not evaluate cognitive and neuropsychiatric complications, c) is strongly influenced by treatment, d) shows a relevant interrater variability [32]. On the other hand, our study of Rep1 SNPs included a larger population (426 patients) with a follow-up of 11.3 years. Progression was measured through clinical milestones, which are reliable measures of patients' status, especially when multicentric studies are concerned.

A second report was published in 2014 by Markopoulou et al [17]. In this study, authors failed to detect a detrimental effect of Rep1 263 allele on PD progression. On the contrary, they found that patients carrying longer Rep1 alleles had a better motor outcome and a similar trend was observed for cognitive outcome, even though without statistical significance. Even in this case, important differences between the two studies might account for the discordant results. Firstly, our clinical data were collected through direct patients' examination whereas cognitive data included in the report by Markopoulou et al. [17] were collected through telephonic interviews. Secondly, the biological effect of Rep1 microsatellite might differ among subjects, especially when a different ethnic background is involved. In fact, Rep1 is a complex microsatellite characterized by a mixed dinucleotide composition. Alleles with the same size could have different composition and it was 
reported that two alleles with the same length but different dinucleotide composition might have different functional effects on the promoter activity [28]. Finally, the way data are presented in the two studies makes results hardly comparable. In fact, Markopoulou et al. [17] aggregate patients carrying two different genotypes (261-261 and 259-263) in a unique very large group, thus losing the impact of 263 allele.

\section{Conclusion}

Our study found a significant and biologically plausible correlation between SNCA Rep1 263 allele and the risk of development of dementia and visual hallucinations in a cohort of Italian PD patients. Future studies on larger patients' populations are mandatory to replicate our findings. Early identification of patients at high risk of complications has relevant implications in terms of both prognosis and disease modifying therapy.

\section{Author roles}

(1) Research project: A. Conception, B. Organization, C. Execution; (2) Statistical Analysis: A. Design, B. Execution, C. Review and Critique; (3) Manuscript: A. Writing of the First Draft, B. Review and Critique

C.L.: 1A, 1C, 3A

D.M.F.: 1B, 1C, 3A

Tu.S.: 2A, 2B

O.G.: $1 \mathrm{C}$

C.M.: 1C

M.L.:1C

Te.S.: $1 \mathrm{C}$

R.G.: $1 C$

D.F.A.: $1 \mathrm{C}, 3 \mathrm{~B}$

L.C.: $1 \mathrm{C}$ 
T.I.: $1 \mathrm{C}$

Z.R.: $1 \mathrm{C}$

Ce.C.: $1 \mathrm{C}$

D.A.S.: $3 B$

M.C.: 2A

C.G.P.: $3 \mathrm{~B}$

B.G.: 3B

P.C.: 1B, 3B

C.R.: 3B

G.S.: $1 B, 3 B$

Co.C.: 1A, 1B, 2C, 3B

Full financial disclosures of all authors, regardless of relationship to current manuscript

This research was supported by the University of Piemonte Orientale, "Fondi di Ricerca Dipartimentale" and by AriSLA (REPEATALS grant).

G.S. received grants from Italian Telethon Foundation: grant n.GTB12001B "Parkinson Institute Biobank" (2012-17). G.S. is coordinator of the Italian consortium of the COURAGE-PD (COmprehensive Unbiased Risk factor Assessement for Genetics and Environment in PD) funded by JPND (The EU Joint Programme - Neurodegenerative Disease Research).

Co. C. received a research grant from the Borghi Foundation, Brebbia (VA), Italy.

\section{References}

[1] Wirdefeldt K, Adami HO, Cole P, Trichopoulos D, Mandel J (2011). Epidemiology and etiology of Parkinson's disease: a review of the evidence. Eur J Epidemiol;26:S1-S58.

[2] Gelb DJ, Oliver E, Gilman S, (1999). Diagnostic criteria for Parkinson disease. Arch Neurol; $56: 33-39$. 
[3] Chaudhuri KR, Odin P, Antonini A, Martinez-Martin P (2011). Parkinson's disease: the nonmotor issues. Parkinsonism Relat Disord; 17:717-723.

[4] De Marchi F, Carecchio M, Cantello R, Comi C (2014). Predicting cognitive decline in Parkinson's disease: can we ask the genes? Front Neurol; 5:224.

[5] Kalia LV, Lang AE (2015). Parkinson's disease. Lancet;386:896-912.

[6] Comi C, Magistrelli L, Oggioni GD, Carecchio M, Fleetwood T, Cantello R, et al (2014). Peripheral nervous system involvement in Parkinson's disease: evidence and controversies. Parkinsonism Relat Disord; 20:1329-1334.

[7] Tofaris GK, Spillantini MG. (2005). Alpha-synuclein dysfunction in Lewy body diseases. Mov Disord; 20:S37-S44.

[8] Cappellano G, Carecchio M, Fleetwood T, Magistrelli L, Cantello R, Dianzani U et al (2013). Immunity and inflammation in neurodegenerative diseases. Am J Neurodegener Dis; 2:89-107.

[9] Chartier-Harlin MC, Kachergus J, Roumier C, Mouroux V, Douay X, Lincoln S, et al (2004) Alpha-synuclein locus duplication as cause of familial Parkinson's disease. Lancet; 364:1167-1169.

[10] Ibáñez P, Bonnet AM, Débarges B, Lohmann E, Tison F, Pollak P et al (2004). Causal relation between alpha-synuclein gene duplication and familial Parkinson's disease. Lancet; 364:1169-1171.

[11] Singleton AB, Farrer M, Johnson J, Singleton A, Hague S, Kachergus J et al (2003). alphaSynuclein locus triplication causes Parkinson's disease. Science;302:841.

[12] Gründemann J, Schlaudraff F, Haeckel O, Liss B. (2008). Elevated alpha-synuclein mRNA levels in individual UV-laser-microdissected dopaminergic substantia nigra neurons in idiopathic Parkinson's disease. Nucleic Acids Res; 36:e38.

[13] Maraganore DM, de Andrade M, Lesnick TG, Strain KJ, Farrer MJ, Rocca WA, et al (2005). High-resolution whole-genome association study of Parkinson disease. Am J Hum Genet;77:685693. 
[14] Maraganore DM, de Andrade M, Elbaz A, Farrer MJ, Ioannidis JP, Krüger R et al (2006). Collaborative analysis of alpha-synuclein gene promoter variability and Parkinson disease. JAMA;296:661-670.

[15] Ritz B, Rhodes SL, Bordelon Y, Bronstein J (2012). $\alpha$-Synuclein genetic variants predict faster motor symptom progression in idiopathic Parkinson disease. PLoS One;7:e36199.

[16] Chung SJ, Biernacka JM, Armasu SM, Anderson K, Frigerio R, Aasly JO et al (2014). $\alpha$ Synuclein repeat variants and survival in Parkinson’s disease. Mov Disord;29:1053-1057.

[17] Markopoulou K, Biernacka JM, Armasu SM, Anderson KJ, Ahlskog JE, Chase BA et al (2014). Does $\alpha$-synuclein have a dual and opposing effect in preclinical vs. clinical Parkinson's disease? Parkinsonism Relat Disord; 20:584-589.

[18] Trotta L, Guella I, Soldà G, Sironi F, Tesei S, Canesi M, et al (2012). SNCA and MAPT genes: Independent and joint effects in Parkinson disease in the Italian population. Parkinsonism Relat Disord;18:257-262.

[19] Berardelli A, Wenning GK, Antonini A, Berg D, Bloem BR, Bonifati V, et al (2013). EFNS/MDS-ES/ENS [corrected] recommendations for the diagnosis of Parkinson's disease. Eur J Neurol; 20:16-34.

[20] Dubois B, Burn D, Goetz C, Aarsland D, Brown RG, Broe GA et al (2007). Diagnostic procedures for Parkinson's disease dementia: recommendations from the movement disorder society task force. Mov Disord; 22:2314-2324.

[21] Comi C, Ferrari M, Marino F, Magistrelli L, Cantello R, Riboldazzi G, et al Polymorphisms of Dopamine Receptor Genes and Risk of L-Dopa-Induced Dyskinesia in Parkinson's Disease. Int J Mol Sci. 2017; 24:18(2). pii: E242. doi: 10.3390/ijms 18020242. 
[22] Ferrari M, Comi C, Marino F, Magistrelli L, De Marchi F, Cantello R, et al. Polymorphisms of dopamine receptor genes and risk of visual hallucinations in Parkinson's patients. Eur J Clin Pharmacol. 2016 Nov;72(11):1335-1341

[23] Zheng J, Yang X, Zhao Q, Tian S, Huang H, Chen Y et al. Festination Correlates with SNCA Polymorphism in Chinese Patients with Parkinson's Disease. Parkinsons Dis. 2017;2017:3176805. doi: $10.1155 / 2017 / 3176805$.

[24] Ffytche DH, Pereira JB, Ballard C, Chaudhuri KR, Weintraub D, Aarsland D. Risk factors for early psychosis in PD: insights from the Parkinson's Progression Markers Initiative. J Neurol Neurosurg Psychiatry. 2017 Apr;88(4):325-331.

[25] Malek N, Swallow DM, Grosset KA, Lawton MA, Marrinan SL, Lehn AC, et al. Tracking Parkinson's: Study Design and Baseline Patient Data. J Parkinsons Dis. 2015;5(4):947-59

[26] Farrer M, Kachergus J, Forno L, Lincoln S, Wang DS, Hulihan M et al (2004). Comparison of kindreds with parkinsonism and alpha-synuclein genomic multiplications. Ann Neurol;55:174-179

[27] Miller DW, Hague SM, Clarimon J, Baptista M, Gwinn-Hardy K, Cookson MR et al (2004). Alpha-synuclein in blood and brain from familial Parkinson disease with SNCA locus triplication. Neurology; 62:1835-1838.

[28] Chiba-Falek O, Nussbaum RL (2001). Effect of allelic variation at the NACP-Rep1 repeat upstream of the alpha-synuclein gene (SNCA) on transcription in a cell culture luciferase reporter system. Hum Mol Genet; 10:3101-3109.

[29] Cronin KD, Ge D, Manninger P, Linnertz C, Rossoshek A, Orrison BM et al (2009). Expansion of the Parkinson disease-associated SNCA-Rep1 allele upregulates human alpha-synuclein in transgenic mouse brain. Hum Mol Genet; 18:3274-3285. 
[30] Fuchs J, Tichopad A, Golub Y, Munz M, Schweitzer KJ, Wolf B, et al (2008) Genetic variability in the SNCA gene influences alpha-synuclein levels in the blood and brain. FASEB J. 22(5):1327-34

[31] Soldner F, Stelzer Y, Shivalila CS, Abraham BJ, Latourelle JC, Barrasa MI et al (2016) Parkinson-associated risk variant in distal enhancer of $\alpha$-synuclein modulates target gene expression. Nature. 5;533(7601):95-9

[32] Grill S, Weuve J, Weisskopf MG. (2011). Predicting outcomes in Parkinson's disease: comparison of simple motor performance measures and The Unified Parkinson's Disease Rating Scale-III. J Parkinsons Dis; 1:287-298. 